\title{
Initial Evaluation of LabPET/SPECT Dual Modality Animal Imaging System
}

Rutao Yao, Senior Member, IEEE, Xiao Deng, Member, IEEE, Jean-François Beaudoin, Tianyu Ma, Member, IEEE, Jules Cadorette, Zixiong Cao, and Roger Lecomte, Member, IEEE

\begin{abstract}
We developed SPECT imaging capability on a LabPET animal scanner to provide an integrated PET/SPECT dual-modality imaging system. The add-on SPECT sub-system was enabled by 1) mechanically integrating a multiple-pinhole collimator in the PET detector ring, and 2) configuring the detectors to acquire singles events in the $120-160 \mathrm{keV}$ range. We report on the design parameters, data acquisition protocols and initial performance assessment of this cost-effective SPECT imaging solution. Phantom and animal images demonstrating the relevance of the system for various imaging tasks in preclinical research are presented.
\end{abstract}

Index Terms-Animal dual-modality imaging, multiple pinhole collimator, PET/SPECT.

\section{INTRODUCTION}

$\mathbf{T}$ O provide a versatile imaging tool for biomedical research, we studied the feasibility of adding a collimator to an existing animal PET scanner so the scanner is also able to perform SPECT imaging. Effectively, this development converts the PET scanner into a dual modality PET/SPECT system [1]. Since the add-on SPECT uses the detector gantry of the animal PET, the main addition required is a collimator insert, whose cost is a small fraction of a stand-alone SPECT system and an insignificant addition to the total cost of the animal PET system.

Manuscript received May 12, 2012; revised September 20, 2012; accepted December 05, 2012. Date of publication January 16, 2013; date of current version February 06, 2013. This work was supported in part by the Pilot Studies program of the University at Buffalo Clinical and Translational Research Center and the Buffalo Translational Consortium, the Canadian Institutes of Health Research (CIHR) Grant MOP-86717, and the Canada Foundation for Innovation (CFI). The Centre de Recherche Clinique Étienne-Le Bel is a FRSQ-funded research center.

R. Yao is with the Department of Nuclear Medicine, State University of New York (SUNY) at Buffalo, Buffalo, NY 14217 USA (e-mail: rutaoyao@buffalo.edu).

X. Deng was with the Department of Nuclear Medicine, SUNY at Buffalo, Buffalo, NY 14217 USA. He is now with the Department of Diagnostic Radiology, Rush University Medical Center, Chicago, IL, 60612 USA (e-mail: xiaodeng@gmail.com).

J.-F. Beaudoin, J. Cadorette, and R. Lecomte are with the Sherbrooke Molecular Imaging Center, Centre de Recherche Clinique Étienne-Le Bel, Centre Hospitalier Universitaire de Sherbrooke, Sherbrooke, QC J1G 2E8, Canada, and the Department of Nuclear Medicine and Radiobiology, Université de Sherbrooke, Sherbrooke, QC J1K 2R1, Canada (e-mail: jf.beaudoin@gmail.com; jules.cadorette@USherbrooke.ca; Roger.Lecomte@USherbrooke.ca).

Z. Cao was with the Department of Nuclear Medicine and Radiobiology, Université de Sherbrooke, Sherbroole, QC J1K 2R1, Canada (e-mail: zixiong@gmail.com).

T. Ma is with the Department of Engineering Physics, Tsinghua University, Beijing 100084, China (e-mail: maty@tsinghua.edu.cn).

Color versions of one or more of the figures in this paper are available online at http://ieeexplore.ieee.org.

Digital Object Identifier 10.1109/TNS.2012.2233216
The proposed dual modality system supports a broad range of imaging applications using both PET and SPECT radiopharmaceuticals, thus achieves a high function-to-cost ratio for investment. In addition, such a dual-modality system has a small overall footprint, and has the potential of enabling PET/SPECT dual tracer research applications. The recent interest in producing both PET and SPECT tracers at a cyclotron facility [2] also enhances the perspective of this integrated PET/SPECT imaging system.

There are several unique technical features involved in the development of the add-on SPECT imaging modality on a PET scanner. The first is a collimator insert needed for performing SPECT imaging. Given that the PET detector gantry is a fixed cylinder, the collimator needs to be designed accordingly to achieve the desired field-of-view (FOV) size and optimal spatial resolution versus sensitivity trade-off. The second unique feature is the use of PET detectors to acquire low-energy, e.g., $140 \mathrm{keV}$, singles photons. The detector settings such as electronics gain and offset, and acquisition energy window, need to be adjusted for the SPECT mode operation. The third feature is that the PET detector crystals are usually pixelated and the crystal layout may not be uniformly distributed [3]. Therefore, special calibration considerations [4] and scan schemes [5] are required for optimal imaging performance.

In this work, we report the initial assessment of the LabPET/SPECT dual modality system. The base animal PET scanner used for the development was a LabPET-8 [3]. The system setup and design specifications are described first. For tomographic imaging, we used a helical scan, which combines collimator rotational movement and subject linear motion. The initial imaging performance of the prototype system is demonstrated with phantom and animal studies.

\section{Materials AND MethodS}

\section{A. Animal PET Scanner}

A LabPET-8 scanner [3] was used for the development. The system consists of 16 detector/ 32 crystal rings. There are 12 cassettes in each detector ring placed in a star-like formation. Each cassette contains 4 detector modules mounted on an analog board. Each module is composed of 4 phoswich detector pairs [6]. A phoswich detector pair has one LYSO and one LGSO scintillator juxtaposed side-by-side axially and coupled to an avalanche photodiode (APD). Each scintillator is $2 \times 2 \times \sim$ $12 \mathrm{~mm}^{3}$ in size. So the scanner has a total of 6144 crystals, with alternating LYSO and LGSO in the axial direction, and phoswich pairs angularly offset by half a crystal size in the azimuthal direction. The inner diameter of the detector ring is 

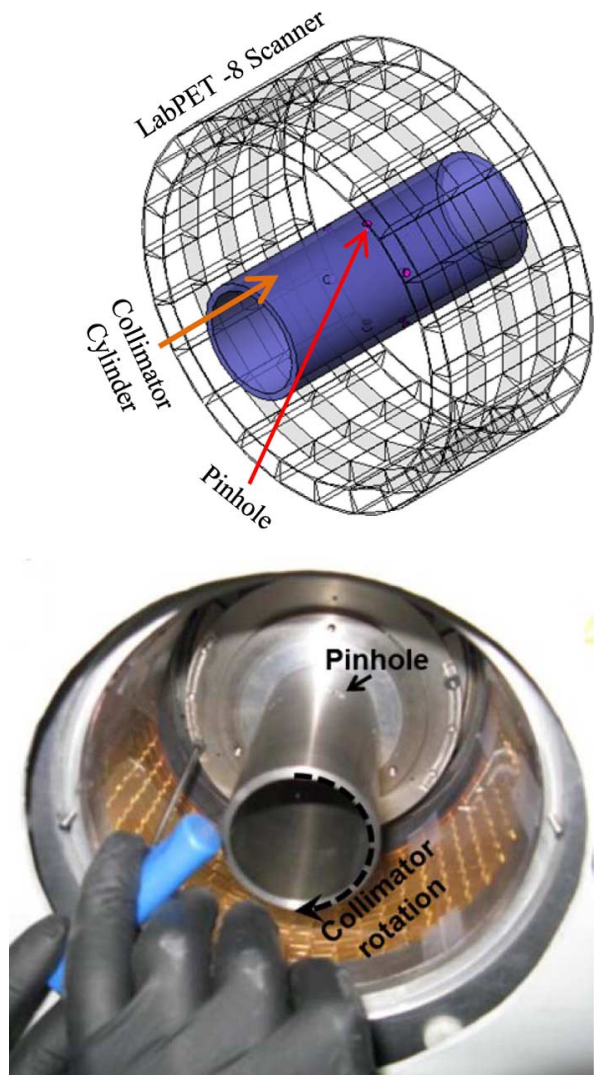

Fig. 1. A schematic drawing (top) and a photo (bottom) showing the 6-pinhole collimator cylinder placed within a LabPET detector ring.

$162 \mathrm{~mm}$ (opening of $150 \mathrm{~mm}$ ) and the axial coverage of the detector is $76 \mathrm{~mm}$.

\section{B. Multiple Pinhole Collimator Insert}

A single-row 6-pinhole collimator was designed as an insert for the add-on SPECT. Given that the PET detector gantry is stationary, and it is undesired to rotate the imaging subject to avoid motion problems, tomographic imaging was achieved by rotating the collimator. A schematic drawing of the pinhole collimator insert within the LabPET-8 detector is shown in Fig. 1. A photo showing the actual pinhole collimator cylinder mounted on the LabPET scanner is also shown in Fig. 1.

The 6-pinhole collimator was designed for mouse imaging studies. The cylinder was made of tungsten ( $90 \%$ purity). Its inner diameter is $44 \mathrm{~mm}$, wall thickness is $3 \mathrm{~mm}$, and length is $90.1 \mathrm{~mm}$. The six pinholes are uniformly distributed on the circle of the middle transverse plane of the cylinder. The diameter and opening angle of the double-knife edged pinhole inserts press-fitted into the cylinder are $1 \mathrm{~mm}$ and $80^{\circ}$, respectively, with a $0.2 \mathrm{~mm}$ high cylindrical central part. The pinhole cylinder assembly is mounted on a rotational holder adapted to the PET rotating source mechanism, as shown in the picture of Fig. 1, for acquiring projections in a tomographic imaging mode [7]. With these geometrical parameters, at the center of FOV (CFOV), the analytically estimated spatial resolution (FWHM) is $1.6 \mathrm{~mm}$ in both tangential and axial directions, and the sensitivity is $0.07 \%$. With collimator rotation only, the shape of the FOV is approximately an ellipsoid with transverse and axial diameters of 30 $\mathrm{mm}$ and $26 \mathrm{~mm}$, respectively.

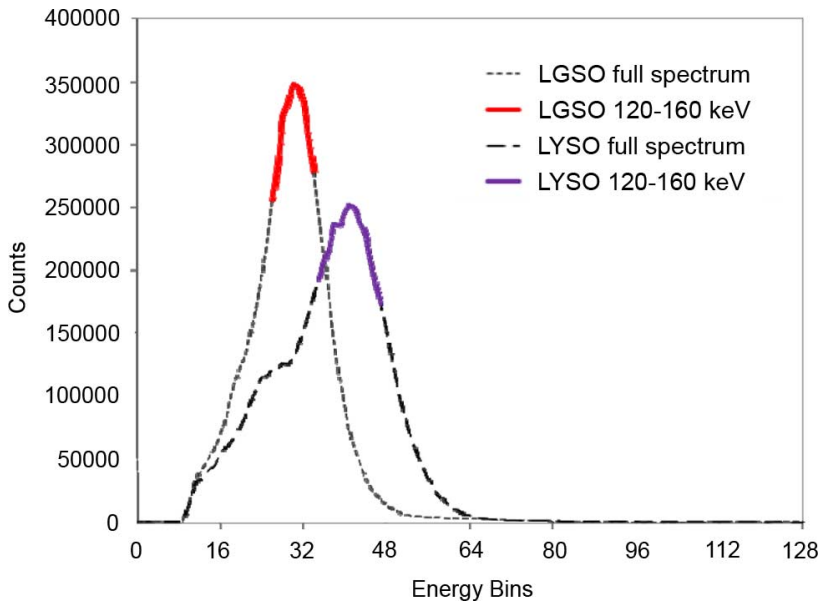

Fig. 2. Sample energy spectra of ${ }^{99 \mathrm{~m}} \mathrm{Tc}$ from the LYSO/LGSO phoswich detector.

\section{Data Acquisition}

Besides mechanical setup, when working in SPECT mode, the LabPET scanner must be configured to acquire singles events with an energy window of 120-160 keV. To achieve this, the detectors are first calibrated per the usual PET mode energy calibration procedure with a ${ }^{68} \mathrm{Ge}$ source. Then, individual detector thresholds are adjusted to ensure that events in the $120-160 \mathrm{keV}$ range are recorded.

Signals from the APD of each phoswich detector pair are amplified and digitized. When a signal's amplitude is above a selected noise threshold, it is accepted as an event and is analyzed - the signal shape is used to identify the scintillating crystal and the signal amplitude is used to represent the energy deposited in the crystal. The event is stored whenever the event's energy falls within the selected energy window, which is $120-160 \mathrm{keV}$ for studies reported in this work. Fig. 2 shows sample energy spectra from the LYSO and LGSO crystals of a phoswich detector pair after crystal discrimination and energy windowing. The estimated energy resolution is $\sim 45 \%$.

The detection efficiency coefficients of the crystals were obtained by scanning a uniform cylindrical phantom filled with $6.19 \mathrm{MBq}{ }^{99 \mathrm{~m}} \mathrm{Tc}$ solution for 25 minutes. The activity volume used was $20.7 \mathrm{~mm}$ diameter $\times 228 \mathrm{~mm}$ length, and was centered at the CFOV. The collimator was removed for this measurement.

With an energy window of 120 to $160 \mathrm{keV}$, the background activity due to the intrinsic radioactivity of ${ }^{176} \mathrm{Lu}$ [8] in the LYSO and LGSO crystals contributes about $0.814 \mathrm{~Bq}$ per crystal to the projection data collected. For each study, this contribution was first estimated based on a separately measured blank (i.e., no object in the FOV) scan and then subtracted [8] from the measured projections. The acquisition time of the blank scan was 5 hours. The collimator was in place during the blank scan to emulate the imaging setup. The same energy window was used for the blank and imaging measurements.

\section{Helical Scan}

To have a uniform and complete projection sampling, we used a helical scan protocol for all imaging studies. The helical protocol is a combination of pinhole cylinder rotation and object 
linear movement. The number of angular steps to complete a full rotation, $N_{r}^{2 \pi}$, to satisfy the angular sampling requirements can be calculated by adapting the standard method described in [9],

$$
R_{\text {pinholeCylinder }} \cdot \frac{2 \pi}{\left(N_{r}^{2 \pi} \cdot N_{\text {pinhole }}\right)}=\frac{\mathrm{FWHM}}{3}
$$

where the radius of FOV $R_{\text {pinholeCylinder }}=15 \mathrm{~mm}$, the number of pinhole $N_{\text {pinhole }}=6$, the estimated spatial resolution at CFOV FWHM $=1.6 \mathrm{~mm}$. The $N_{r}^{2 \pi}$ calculated is 30 , which corresponds to 2 degrees per angular-step.

The axial step size $S_{a x}$ we used was $0.6 \mathrm{~mm}$, which is approximately $1 / 3$ of the axial FWHM. Whereas the axial step size can be further optimized for image quality or imaging speed, the chosen value was found suitable for the initial demonstration of the SPECT imaging capability.

The typical acquisition sequence includes the following steps: 1) acquire data for a pre-determined time, e.g., 180 seconds, at its starting position; 2) move the object linearly with step size of $S_{a x}=0.6 \mathrm{~mm}$ in axial direction; 3 ) rotate the pinhole cylinder for $\theta=2$ degrees; and 4 ) repeat the last 3 steps $N_{r}$ times to ensure proper angular and axial sampling across the entire $N_{r} \times S_{a x} \mathrm{~mm}$ axial length of FOV. In the following context, this helical scan is described as $N_{r} \times\left[\theta, S_{a x}, t_{a c q}\right]$, where $t_{a c q}$ is the acquisition time after each helical movement. The axial FOV can be extended by adding additional helical steps to encompass the desired region-of-interest of the imaged object.

To accumulate firsthand experience on the performance of different settings, we experimented with different helical settings.

\section{E. Image Generation}

The image generation protocol we used consists of 3 steps: 1) detector intrinsic background activity correction, 2) detector sensitivity normalization, 3) iterative statistical image reconstruction.

The detector efficiency coefficients were applied to the projection data pre-corrected for ${ }^{176} \mathrm{Lu}$ background and radioactive decay. Scatter and attenuation corrections were not implemented at this stage. Due to the small size of the targeted imaging objects with this system, i.e., mice, these corrections are expected to have negligible effects on image quality. Since all the imaging studies were completed in less than 2.5 hours, the effect of radioactive decay was relatively small. Decay correction was not applied to the projection data presented here but will be in the future.

An iterative reconstruction program with the standard ordered subset expectation maximization (OSEM) algorithm [10] was used for image reconstruction. To generate accurate system response matrix, the geometrical calibration was carried out first using an analytical method described previously [7].

The system matrix used for image reconstruction consisted of sub-system matrices of the pinhole collimator at each rotation position, as each rotation of the pinhole cylinder creates a new detector configuration, which corresponds to a different image system response function that needs to be modeled by a new system matrix. This means the system matrix is $n$ times that of the stationary collimator setup, where $n$ is the number of rotation steps. An accurate and compact system matrix modeling method was developed to overcome this challenge [11]

The parameters used in the OSEM reconstruction software reported in this work, unless described specifically, was 10 subsets and 5 iterations.

\section{F. Phantom and Animal Imaging Studies}

The imaging performance of the system was assessed using two phantoms. An Ultra-Micro Hot-Rod phantom (Data Spectrum Inc., Hillsborough, NC) was filled with 86.6 MBqof a ${ }^{99 \mathrm{~m}} \mathrm{Tc}$-pertechnetate solution to investigate the transaxial resolution. The phantom was scanned with a helical protocol of $30 \times\left[2^{\circ}, 0.45 \mathrm{~mm}, 60\right.$ seconds $]$. A modified Defrise phantom consisting of a stack of 5 discs of increasing thicknesses from 2.3 to $3.2 \mathrm{~mm}$ was then scanned to assess the axial resolution. The discs were spaced with the same distance as the disc thickness. It was filled with $149.5 \mathrm{MBq}{ }^{99 \mathrm{~m}} \mathrm{Tc}$-pertechnetate solution and scanned with a $60 \times\left[2^{\circ}, 0.6 \mathrm{~mm}, 60\right.$ seconds $]$ helical protocol.

The mouse imaging capability of the system was then investigated in three different studies. First, a whole body bone scan using ${ }^{99 \mathrm{~m}} \mathrm{Tc}-\mathrm{MDP}$ was performed. The weight of the mouse was $23.7 \mathrm{~g}$ and the injected dose was $172.3 \mathrm{MBq}$. The helical protocol was $270 \times\left[2^{\circ}, 0.2 \mathrm{~mm}, 30\right.$ seconds]. The scan was started at 2 hour and 45 minutes after radiotracer injection.

To investigate the ability of the pinhole SPECT system to image targeted organs, a mouse cardiac imaging study was then performed. A 21.4 gram HFS mouse (female) was injected with $36.7 \mathrm{MBq}$ of ${ }^{99 \mathrm{~m}} \mathrm{Tc}-$ Sestamibi and scanned starting at 74 minutes post-injection. The scan protocol was $30 \times\left[2^{\circ}, 0.0 \mathrm{~mm}, 60\right.$ seconds $]$, the $0.0 \mathrm{~mm}$ linear step size indicates that only rotation of the collimator was used.

Finally, the system was used to compare the imaging properties of ${ }^{99 \mathrm{~m}} \mathrm{TcO}_{4}{ }^{-}$produced by a cyclotron [2] and by a standard ${ }^{99} \mathrm{Mo} /{ }^{99 \mathrm{~m}} \mathrm{Tc}$ generator. One mouse $(17 \mathrm{~g})$ was injected with $8.8 \mathrm{MBq}$ of cyclotron-produced ${ }^{99 \mathrm{~m}} \mathrm{Tc}$ by the ${ }^{100} \mathrm{Mo}(\mathrm{p}, 2 \mathrm{n})^{99 \mathrm{~m}} \mathrm{Tc}$ reaction, while the other mouse $(26.8$ g) was injected with $9.3 \mathrm{MBq}$ of the ${ }^{99 \mathrm{~m}} \mathrm{Tc}$ eluted from a standard ${ }^{99} \mathrm{Mo} /{ }^{99 \mathrm{~m}} \mathrm{Tc}$ generator. The helical scan protocol was $30 \times\left[2^{\circ}, 0.2 \mathrm{~mm}, 208\right.$ seconds $]$. The targeted imaging organ of the study was the thyroid and the aim was to demonstrate that ${ }^{99 \mathrm{~m}} \mathrm{Tc}$-pertechnetate produced by cyclotron or eluted from a ${ }^{99} \mathrm{Mo} /{ }^{99 \mathrm{~m}} \mathrm{Tc}$ generator yielded the same diagnostic information.

\section{RESULTS}

\section{A. Hot-Rod Phantom Study}

Fig. 3 shows the summed image of 28 transaxial slices of the Ultra-Micro Hot-Rod phantom. The $1 \mathrm{~mm}$ hot rods can be resolved in this image, indicating that the expected system FWHM spatial resolution of $1.6 \mathrm{~mm}$ is clearly reached and even exceeded.

\section{B. Modified Defrise Phantom}

The coronal view of the modified Defrise phantom displayed in Fig. 4 shows a clear separation of the hot discs. This is 


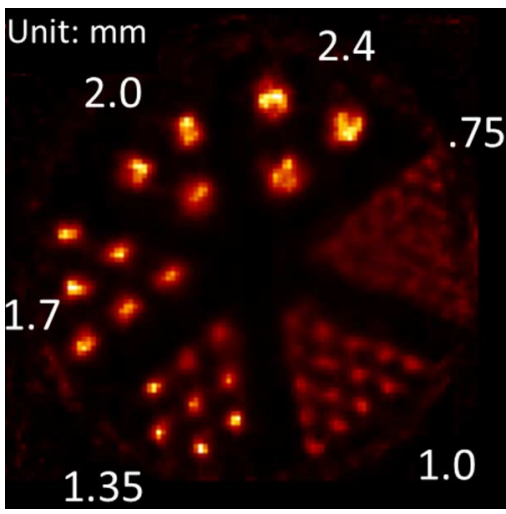

Fig. 3. Transverse view image of the Ultra-Micro Hot-Rod phantom. The distance between centers of neighboring hot-rods is twice the hot-rod diameters indicated in the image. The image shown is the sum of 28 slices $(8.4 \mathrm{~mm}$ in the axial direction), and was obtained with 20 OSEM iterations.

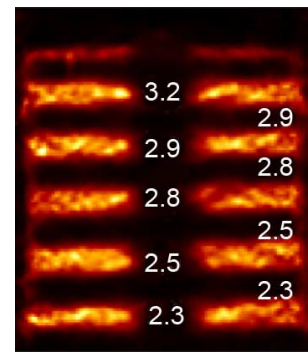

Fig. 4. Coronal view of the modified Defrise phantom acquired with a helical scan. The numbers indicate the thicknesses, in $\mathrm{mm}$, of the alternating plastic and activity discs.

expected because the designed axial resolution (FWHM) is $1.6 \mathrm{~mm}$, which is higher than what is needed to distinguish the $2.3 \mathrm{~mm}$ and thicker discs. This shows that the helical scan settings used are adequate for extending the axial imaging volume. The top activity disc in the image is partially missing due to incomplete axial coverage of the helical scan-the pinhole plane must be stepped beyond the intended axial image volume for complete data sets.

\section{Mouse Bone Study}

Fig. 5 shows the image of the mouse bone scan with ${ }^{99 \mathrm{~m}} \mathrm{Tc}-\mathrm{MDP}$. The vertebrae and main joints of the mouse can be clearly resolved. The small axial step size $(0.2 \mathrm{~mm})$ used for this acquisition limited the axial image volume, so the skull and the tail-bone of the mouse were not imaged properly. The image definition demonstrated here shows that the add-on SPECT resolution performance is suitable for mouse studies.

\section{Mouse Cardiac Study}

The transverse images of the mouse heart in multiple planes are shown in Fig. 6. The clear donut shape of the left myocardium indicates that the resolution is adequate for mouse cardiac studies. As the mouse heart is only about $10 \mathrm{~mm}$ in any dimension, the rotation-only scan protocol may be used, but further improvements on detector configuration and evaluation of image quality will be needed to ensure that no image distortion or under-sampling artifact is present. It would be straight forward to introduce gated imaging for measurement of the ventricular volumes and ejection fraction.

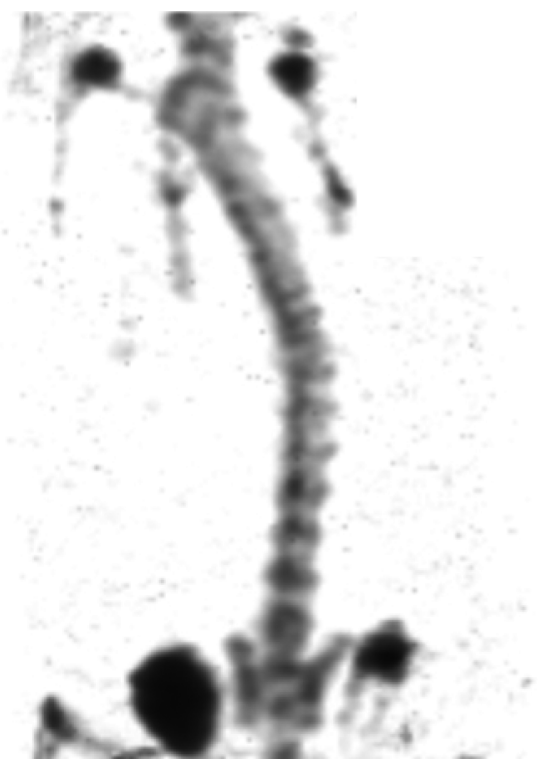

Fig. 5. MIP (maximum intensity projection) view of a ${ }^{99 \mathrm{~m}} \mathrm{Tc}-\mathrm{MDP}$ mouse bone scan study acquired with the pinhole collimator.
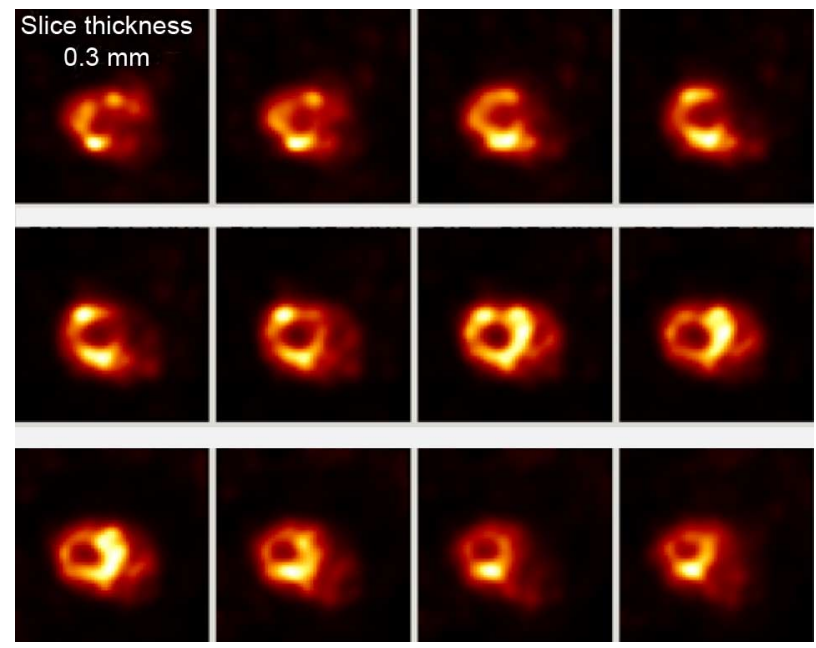

Fig. 6. Transverse views across the in vivo heart of a 21-gram mouse (nongated).

\section{E. Mouse Thyroid Study}

The thyroid uptake of ${ }^{99 \mathrm{~m}} \mathrm{TcO}_{4}^{-}$produced by a cyclotron is compared to that of ${ }^{99 \mathrm{~m}} \mathrm{TcO}_{4}^{-}$produced by a generator in two different mice. The thyroids in both mice could be clearly identified and both images showed normal thyroid uptake. The background pattern in the left image, ${ }^{99 \mathrm{~m}} \mathrm{TcO}_{4}^{-}$from cyclotron, seems to be slightly higher than that in the right image. It is unclear this was due to difference in ${ }^{99 \mathrm{~m}} \mathrm{TcO}_{4}^{-}$or in the mice.

\section{DISCUSSION}

The stationary PET detector ring poses challenges as well as opportunities for SPECT development. One challenge is that given the desired FOV size for mouse imaging, the magnification factor attainable is only about 2.5 . As a result, the achievable spatial resolution is limited by the detector's intrinsic resolution, which is determined by the finite size of the PET detector elements. Consequently, the PET-based pinhole SPECT approach cannot reach as high spatial resolution as dedicated 


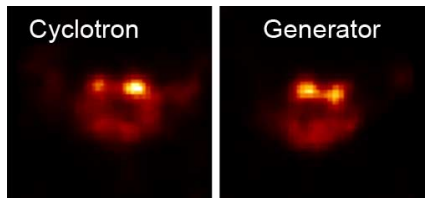

Fig. 7. Comparison of mouse thyroid uptakes of ${ }^{99 \mathrm{~m}} \mathrm{TcO}_{4}^{-}$produced by cyclotron and by generator.

small animal SPECT systems [12]-[15]. Another challenge is the irregular spacing of the PET detectors, which imposes an accurate modeling of the detector response for calculating the system matrix and proper sampling schemes for complete data sets. A third challenge relates to the need of reconfiguring the PET detectors and data acquisition system to acquire singles events in an energy window of $\sim 120-160 \mathrm{keV}$. The fully digital data acquisition system of the LabPET ${ }^{\mathrm{TM}}$ easily allows such settings [6]. However, calibrating the PET detectors for measurement of lower energy radiation requires revision of the operating conditions, including avalanche photodiode (APD) bias and crystal identification threshold in some cases, to reduce electronic noise and improve signal-to-noise ratio in this lower energy range. Such optimization was not carried out systematically in the present implementation and would deserve more attention for optimal detector characteristics in the future. In spite of those limitations, the overall SPECT performance was shown to be adequate and reliable for a large range of mouse imaging applications. It is worth noticing that the achieved SPECT spatial resolution is comparable to that achieved in PET mode with the LabPET ${ }^{\mathrm{TM}}$ system [3], which would facilitate PET/SPECT dual-modality imaging.

We reported previously [8] that, with an energy window of 135 to $145 \mathrm{keV}$, the average count rate per crystal from LSO crystal intrinsic activity was $0.042 \mathrm{~Bq}$ on a microPET Focus 120 scanner. Even taking into account the energy window width used then was only a quarter of that used in this study, the 0.814 $\mathrm{Bq}$ per crystal background count measured on LabPET now is significantly higher. Therefore it is very desirable to optimize the configuration of the PET detectors and data acquisition system for acquiring singles events in the SPECT energy window with maximum efficiency and minimum background count rate. On the other hand, the imaging studies presented show that the template based background correction method [8] is proven to be also effective with very low average count rate from the imaged subject. For example, in the mouse cardiac study, the net count rate per crystal was about $0.012 \mathrm{~Bq}$, less than $2 \%$ of the background count rate. This study also shows that the background correction method works well with phoswich detectors.

The major opportunity offered by the stationary PET detector ring is that SPECT imaging capability can be implemented with a simple compact rotational collimatorat a tiny fraction of the cost of a dedicated SPECT system. The small capital investment also provides flexibility of utilizing different collimators for different in vivo imaging applications. Rotation of the collimator, which has been mounted on the existing rotating source holder and was activated using the control software of the PET scanner, is also easier to implement than the rotation of the full detector assembly of most dedicated small animal SPECT system. Another advantage of a full detector ring is that a full data set can be collected with a partial rotation of only $60^{\circ}$ with the current 6-pinhole collimator. PET imaging with the collimator in place is another key opportunity that can be utilized for geometrical calibration of the SPECT device [16]. The collimator center-of-rotation and exact pinhole positions can indeed be measured accurately using strategically positioned positron point or line sources on the pinhole collimator cylinder.

Some of the helical scan parameters used in this work needs optimization. For example, we found that the axial volume defined by the starting and ending pinhole planes [17] is not completely sampled at the two ends of the volume, where the image volume reconstructed shows streak artifacts. The axial step size, as a critical factor impacting total scan time, may be somewhat increased without compromising image quality. In addition, the rotational angular step size may also be increased owing to the projection sampling in the oblique planes, which is further enhanced by the staggered detector design of the LabPETTM, where neighboring detector rings are angularly offset by one half-crystal. The optimal choice of parameters for a given imaging task requires further investigation [18].

\section{SUMMARY}

We have developed SPECT imaging capability on a LabPET-8 scanner with a pinhole collimator insert. Phantom and animal studies with the pinhole collimator are presented in this work and have demonstrated volume imaging capability with image resolution close to the system design specifications and comparable to the PET imaging resolution.

\section{ACKNOWLEDGMENT}

The authors would like to acknowledge the help of Véronique Dumulon-Perreault and Michel Paquette in preparing and handling the animals used in this study. This work utilized the highperformance computational capabilities of the Linux cluster at the Center for Computational Research, State University of New York at Buffalo.

\section{REFERENCES}

[1] T. Ma, Y. Shao, M. Sajjad, H. A. Nabi, and R. Yao, "SPECT mouse imaging on a hybrid micro-PET/SPECT system," J. Nucl. Med., vol. 49, p. 119P, 2008.

[2] B. Guerin, S. Tremblay, S. Rodrigue, J. A. Rousseau, V. Dumulon-Perreault, and R. Lecomte et al., "Cyclotron production of ${ }^{99_{\mathrm{m}} \mathrm{Tc}} \mathrm{T}$ : An approach to the medical isotope crisis," J. Nucl. Med., vol. 51, pp. $13 \mathrm{~N}-16$, Apr. 2010.

[3] M. Bergeron, J. Cadorette, J. F. Beaudoin, M. D. Lepage, G. Robert, and V. Selivanov et al., "Performance evaluation of the LabPET APDbased digital PET scanner," IEEE Trans. Nucl. Sci., vol. 56, pp. 10-16, Feb. 2009

[4] X. Deng, T. Ma, R. Lecomte, and R. Yao, "Optimization and calibration of slat position for a SPECT with slit-slat collimator and pixelated detector crystals," IEEE Trans. Nucl. Sci., vol. 58, no. 5, pp. 2234-2243, Oct. 2011

[5] Y. Xia, T. Ma, X. Deng, Y. Liu, Y. Jin, and S. Murali et al., "A hybrid rotation-translation scan scheme for in vivo animal SPECT imaging on a stationary scanner," in Proc. IEEE Nucl. Sci. Symp. Conf. Rec., Orlando, FL, 2009, pp. 2686-2689.

[6] R. Fontaine, F. Belanger, N. Viscogliosi, H. Semmaoui, M. A. Tetrault, and J. B. Michaud et al., "The hardware and signal processing architecture of LabPET (TM), a small animal APD-based digital PET scanner," IEEE Trans. Nucl. Sci., vol. 56, no. 1, pp. 3-9, Feb. 2009. 
[7] X. Deng, T. Ma, J. Cadorette, Z. Cao, J.-F. Beaudoin, and R. Lecomte et al., "Geometrical calibration for an animal PET converted SPECT," in Proc. IEEE Nucl. Sci. Symp. and Med. Imag. Conf. Rec., Knoxville, TN, 2010, pp. 3038-3041.

[8] R. Yao, T. Ma, and Y. Shao, "Lutetium oxyorthosilicate (LSO) intrinsic activity correction and minimal detectable target activity study for SPECT imaging with a LSO-based animal PET scanner," Phys. Med. Biol., vol. 53, pp. 4399-4415, 2008.

[9] S. R. Cherry, J. A. Sorenson, and M. E. Phelps, Physics in Nuclear Medicine, 3rd ed. Philadelphia, PA: Saunders, 2003.

[10] H. M. Hudson and R. S. Larkin, "Accelerated image reconstruction using ordered subsets of projection data," IEEE Trans. Med. Imag., vol. 13, no. 4, pp. 601-609, Dec. 1994.

[11] T. Ma, X. Deng, R. Lecomte, and R. Yao, "Derivation of the system matrix for an animal SPECT scanner with rotational collimator and stationary ring detector," in Proc. IEEE Nucl. Sci. Symp. Med. Imag. Conf. Rec., Knoxville, TN, 2010, pp. 2288-2291.

[12] F. J. Beekman, F. van der Have, B. Vastenhouw, A. J. A. van der Linden, P. P. van Rijk, and J. P. H. Burbach et al., "U-SPECT-I: A novel system for submillimeter-resolution tomography with radiolabeled molecules in mice," J. Nucl. Med., vol. 46, pp. 1194-1200, Jul. 2005.
[13] F. v. d. H. Vastenhouw et al., "U-SPECT-II: An ultra-high-resolution device for molecular small-animal imaging," J. Nucl. Med., vol. 50, pp. 599-599, 2009.

[14] T. Funk, P. Despres, W. C. Barber, K. S. Shah, and B. H. Hasegawa, “A multipinhole small animal SPECT system with submillimeter spatial resolution," Med. Phys., vol. 33, pp. 1259-1268, May 2006.

[15] B. L. Franc, P. D. Acton, C. Mari, and B. H. Hasegawa, "Small-animal SPECT and SPECT/CT: Important tools for preclinical investigation," J. Nucl. Med., vol. 49, pp. 1651-1663, Oct. 2008.

[16] X. Deng, J. F. Beaudoin, J. Cadorette, M. Tianyu, R. Lecomte, and Y. Rutao, "Slit-slat collimator geometrical calibration for a PET/SPECT dual modality animal scanner," in Proc. IEEE Nucl. Sci. Symp. Med. Imag. Conf. Rec., Valencia, Spain, 2011, pp. 2439-2442.

[17] S. D. Metzler, S. Vemulapalli, R. J. Jaszczak, G. Akabani, and B. B. Chin, "Feasibility of whole-body functional mouse imaging using helical pinhole SPECT," Molec. Imag. Biol., vol. 12, pp. 35-41, Feb. 2010.

[18] T. Dai, T. Ma, Q. Wei, S. Wang, H. Liu, and J. Cui et al., "Design and sampling completeness evaluation of scanning orbits in multi-pinhole small animal SPECT imaging," in Proc. IEEE Nucl. Sci. Symp. Med. Imag. Conf. Rec., Valencia, Spain, 2011, pp. 2836-2839. 